\title{
Medicinal and Economic Values of Forest Products in the Treatment of Cancer in Southwest Nigeria
}

\author{
Oluwakemi Osunderu* \\ Federal College of Complementary and Alternative Medicine, Nigeria
}

Submission: August 11, 2017; Published: September 15, 2017

*Corresponding author: Oluwakemi Osunderu, Federal College of Complementary and Alternative Medicine, Nigeria, Email: oaoluwakemi@gmail.com

\begin{abstract}
Medicinal plants are used to address the twin problems of promoting sustainable livelihoods and treatment of numerous illnesses in Nigeria. The study examined the medicinal value of forest products in the treatment of cancer in South-west Nigeria. Primary data was obtained in a cross section survey of 327 respondents comprising 127 Traditional Medicine Practitioners (TMPs), 100 Orthodox Medicine Practitioners (OMPs) and 100 respondents from the General Public drawn by multistage sampling technique from the study area. Interview schedule was used in collection of data on the effectiveness of forest products in cancer treatment. The result showed that seven species were identified belonging to seven different families; Rutaceae, Asteraceae, Anarcardiaceae, Annonaceae, Meliaceae, Guttiferaceae and Leguminaceae topped the TMPs priority list. Result of economic analysis shows minimal competition in the anti-cancer forest product market and a high level of monopoly with a Gini coefficient of 0.83 .

The rate of return on investment was $180.08 \%$ indicating that the TMPs were making profit. Five of the plants were tested against cancer cell lines MCF7 and Hs578T while Doxorubicin (a synthetic anticancer drug) was used as the control treatment. Three plants; Saccharum offinarum (Stem), Sucurinega virosa (Root) and Piper guineensii (Seed) produced no result; Garcinia kola (Bark) did not exhibit any anticancer effect even at a concentration of $10 \mathrm{u} 1 / \mathrm{m} 1$ while only one plant species was effective against the cancer cell line at $1 \mathrm{u} 1 / \mathrm{m} 1$. It is therefore concluded that forest products are effective in the treatment of cancer.
\end{abstract}

Keywords: Medicinal plants; Cancer; Traditional medicine practitioners (Tmps); Forest products; Southwest Nigeria

\section{Introduction}

Medicinal plants are important for a number of reasons. A large proportion of the world's rural population depends on these plants for their health care needs Largo [1]. They also provide the basic raw material for the production of traditional medicines FAO [2], FAO [3]. The collection and processing of medicinal plants provide employment and income opportunities for a large number of people in rural areas Marshall et al. [4].

The importance of traditional medicinal plants in conservation of biological diversity also merits attention Okoli [5]. WHO has been conducting studies on medicinal plants. These studies prompted the initial identification of 20000 species of medicinal plants and a more detailed investigation of a short list of 200 (WHO [6], WHO [7]. Olopade [8] and Odugbemi [9] reported that a great number of these plants have their origins in the world's tropical forests and their present use is largely rooted in traditional medicines which play a major part in maintaining the health and welfare of both rural and city dwellers in developing countries.

More than $60 \%$ of world's total new annual cases occur in Africa, Asia and Central and South America. These regions account for $70 \%$ of the world's cancer deaths. It is expected that annual cancer cases will rise from 14 million in 2012 to 22 million within the next two decades IARC [10], WHO [11]. Consequently, there is need to institute measures that will ensure the availability of anticancer forest products in the forest of Southwest Nigeria and ensure the sustainability of the practice of the TMPs who use forest products to treat cancer. It has been estimated that as many as $75 \%$ to $90 \%$ of the world's rural people rely on herbal traditional medicine as their primary health care WHO [7] and this is a source of income for the growers of such plants and the TMPs USAID [12]. African flora is potential for new compounds with pharmacological activities. Such efforts have led to the isolation of several biologically active molecules that are in various stages of development as pharmaceuticals.

The main objective of this study is to evaluate the economic and medicinal value of forest products in the treatment of cancer in southwest Nigeria, particularly Ogun State and the specific objectives are:

a. To determine the availability of medicinal plants used for the treatment of cancer in Southwest Nigeria.

b. To determine the efficacy of some of the forest products used for the treatment of cancer in Southwest Nigeria.

c. To investigate the stakeholders' socioeconomic 
characteristics and their involvement in the usage of forest products for the treatment of cancer in Southwest Nigeria.

d. To determine the factors that affect the income of the TMPs in the study area and the market structure of forest products used for the treatment of cancer in Southwest Nigeria.

\section{Sampling method, sample selection and data collection}

Data sources and collection: For the purpose of data collection in this study, field trips, collection of available medicinal plant species used for the treatment of cancer, determination of their species type, oral interviews of Traditional Medicine Boards officials, administration of structured questionnaires on relevant target groups, that is, Traditional Medicine Practitioners (TMPs), Orthodox Medicine Practitioners (OMPs) and the General Public (GP) were carried out. Ethno medicinal surveys were also conducted in the study area for collection of data related to the medicinal use of forest products in the treatment of cancer in addition to the pharmacological screening of the plants to determine the level of their efficacy in the treatment of cancer and to validate the claims of the TMPs (Figure 1).

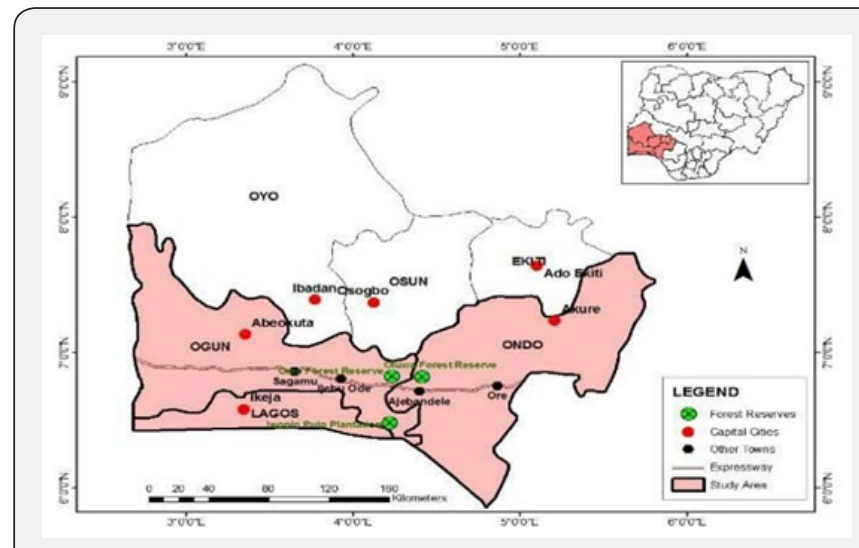

Figure 1: Map of Southwest Nigeria Inset: Lagos and Ogun States.

To identify the locations with high concentration of TMPs in the Study Area, primary data were obtained through oral interviews of the officials of the Hospital Management Department of the Federal Ministry of Health, Federal college of Complementary and Alternative Medicine (FEDCAM), Abuja and the Nigeria Natural Medicine Development Agency, Lagos. Multistage sampling technique was employed. The South

Western Nigeria was first stratified into six states to produce primary units namely: Ekiti, Lagos, Ogun, Ondo, Osun and Oyo. Out of these primary units, Ogun State was purposively sampled because of the high concentration of TMPs in the State.

Results

\section{Availability of medicinal plants used for the treatment of cancer in South-Western Nigeria}

List of Plants Used by the Traditional Medicine Practitioners in the Treatment of Cancer. Estimated Cost Range = 500$10,000 \mathrm{Naira} / \mathrm{kg}$

\begin{tabular}{|c|c|c|c|c|c|c|c|c|c|}
\hline \multicolumn{10}{|c|}{ List of Plants Used by the Traditional Medicine Practitioners in the Treatment of Cancer. Estimated Cost Range $=500-10,000$ Naira/kg } \\
\hline S/No & $\begin{array}{l}\text { Local } \\
\text { Name }\end{array}$ & Species & Family & $\begin{array}{l}\text { Floral } \\
\text { Type }\end{array}$ & Source & $\begin{array}{c}\text { Status of } \\
\text { availability }\end{array}$ & Parts used & Form used & Products \\
\hline \multirow[b]{2}{*}{1} & \multirow{2}{*}{ Eru } & Xylopia aethiopica & \multirow{2}{*}{ Annonaceae } & \multirow{2}{*}{ Tree } & \multirow{2}{*}{ Free areas } & \multirow{2}{*}{ Abundant } & \multirow{2}{*}{$\begin{array}{l}\text { Fruit, } \\
\text { branches }\end{array}$} & \multirow{2}{*}{$\begin{array}{c}\text { Green, dry } \\
\text { (Water boiled). }\end{array}$} & \multirow{2}{*}{$\begin{array}{l}\text { Firewood, } \\
\text { Medicinal }\end{array}$} \\
\hline & & (Dunal) A. Rich & & & & & & & \\
\hline \multirow[t]{2}{*}{2} & \multirow[t]{2}{*}{ Oganwo } & \multirow[t]{2}{*}{ Khaya ivorensisA Chev } & \multirow[t]{2}{*}{ Meliaceae } & \multirow[t]{2}{*}{ Tree } & \multirow[t]{2}{*}{ Free areas } & \multirow[t]{2}{*}{ Rare } & $\begin{array}{c}\text { Stem, } \\
\text { Branches }\end{array}$ & \multirow[t]{2}{*}{ Dry } & \multirow{2}{*}{$\begin{array}{l}\text { Firewood, } \\
\text { Medicinal }\end{array}$} \\
\hline & & & & & & & Bark & & \\
\hline 3 & Mango & Magnifera indicaLinn & Anacardiaceae & Fruit Tree & $\begin{array}{l}\text { Free areas, } \\
\text { Forest, } \\
\text { plantation }\end{array}$ & Abundant & $\begin{array}{l}\text { Leaves, } \\
\text { fruits, bark, } \\
\text { branches, } \\
\text { stem }\end{array}$ & $\begin{array}{c}\text { Green, } \\
\text { dry(Water } \\
\text { boiled ). }\end{array}$ & $\begin{array}{c}\text { Fruit, } \\
\text { firewood, } \\
\text { medicinal }\end{array}$ \\
\hline 4 & Kaju & Anacardium occidentalisLinn & Anacardiaceae & Fruit Tree & $\begin{array}{l}\text { Free areas, } \\
\text { Farmland, } \\
\text { forest, } \\
\text { plantation }\end{array}$ & Abundant & $\begin{array}{l}\text { Fruits, } \\
\text { branches, } \\
\text { stem }\end{array}$ & $\begin{array}{c}\text { Green, } \\
\text { dry(Water } \\
\text { boiled ). }\end{array}$ & $\begin{array}{c}\text { Fruit, } \\
\text { firewood, } \\
\text { medicinal }\end{array}$ \\
\hline 5 & Iyeye & Spondias mombinLinn & Anacardiaceae & Fruit Tree & $\begin{array}{c}\text { Farmland, } \\
\text { Free areas, } \\
\text { forest }\end{array}$ & Abundant & Fruits, bark & $\begin{array}{c}\text { Green, } \\
\text { dry(Water } \\
\text { boiled ). }\end{array}$ & $\begin{array}{c}\text { Fruit, } \\
\text { medicinal }\end{array}$ \\
\hline 6 & Abo & AnnonasenegalensisPers & Annonaceae & Shrub & $\begin{array}{l}\text { Free areas, } \\
\text { forest }\end{array}$ & Abundant & $\begin{array}{c}\text { Leaves, } \\
\text { fruits, stem }\end{array}$ & $\begin{array}{c}\text { Green, } \\
\text { dry(Water } \\
\text { boiled ). }\end{array}$ & $\begin{array}{l}\text { Medicinal, } \\
\text { fruit, } \\
\text { firewood }\end{array}$ \\
\hline
\end{tabular}


Journal of Tumor Medicine \& Prevention

\begin{tabular}{|c|c|c|c|c|c|c|c|c|c|}
\hline 7 & Ahun & $\begin{array}{c}\text { Alstoniaboonei } \\
\text { De Wild }\end{array}$ & Apocynaceae & Tree & $\begin{array}{l}\text { Free areas, } \\
\text { forest }\end{array}$ & Scarce & $\begin{array}{l}\text { Leaves, bark, } \\
\text { root }\end{array}$ & $\begin{array}{c}\text { Green, } \\
\text { dry(Powder) }\end{array}$ & $\begin{array}{c}\text { Medicinal, } \\
\text { firewood }\end{array}$ \\
\hline 8 & Osanwewe & Citrus medicaLinn. & Rutaceae & Shrub & $\begin{array}{l}\text { Free areas, } \\
\text { forest }\end{array}$ & Abundant & Leaves & $\begin{array}{c}\text { Green, } \\
\text { dry(Water } \\
\text { boiled ). }\end{array}$ & Medicinal \\
\hline 9 & Oruwo & MorindalucidaBenth. & Rubiaceae & Tree & $\begin{array}{l}\text { Free areas, } \\
\text { forest }\end{array}$ & Abundant & Leaves & $\begin{array}{c}\text { Green, dry(Cold } \\
\text { water squeezed } \\
\text { ). }\end{array}$ & Medicinal \\
\hline 10 & Oori-nla & VitexdonianaSweet & Verbenaceae & Tree & $\begin{array}{l}\text { Free areas, } \\
\text { forest }\end{array}$ & Abundant & Fruit, leaves & $\begin{array}{l}\text { Green(Water } \\
\text { boiled ). }\end{array}$ & $\begin{array}{l}\text { Fruit, } \\
\text { medicinal }\end{array}$ \\
\hline 11 & Osopupa & EnantiachloranthaOliv. & Annonaceae & Tree & $\begin{array}{l}\text { Free areas, } \\
\text { forest }\end{array}$ & Abundant & Bark & $\begin{array}{c}\text { Green, } \\
\text { dry(Water } \\
\text { boiled ). }\end{array}$ & Medicinal \\
\hline 12 & Owu-elepa & PiliostigmathinningiMilne Redhead & $\begin{array}{l}\text { Leguminosae Sub: } \\
\text { Mimosoidae }\end{array}$ & Shrub & $\begin{array}{l}\text { Free areas, } \\
\quad \text { forest }\end{array}$ & Abundant & Leaves & $\begin{array}{c}\text { Green, } \\
\text { dry(Water } \\
\text { boiled ). }\end{array}$ & Medicinal \\
\hline 13 & Putu & Ricinodendronheudelotii(Baill) Heckel & Euphorbiaceae & Tree & $\begin{array}{l}\text { Free areas, } \\
\text { forest }\end{array}$ & Abundant & Leaves, bark & $\begin{array}{c}\text { Green, } \\
\text { dry(Water } \\
\text { boiled ). }\end{array}$ & Medicinal \\
\hline 14 & Opoto & FicussurForssk. & Moraceae & Tree & $\begin{array}{l}\text { Free areas, } \\
\text { forest }\end{array}$ & Abundant & Fruit, bark & $\begin{array}{c}\text { Green, } \\
\text { dry(Water } \\
\text { boiled ). }\end{array}$ & $\begin{array}{l}\text { Fruit, } \\
\text { medicinal }\end{array}$ \\
\hline 15 & Asasa & Margaritariadiscoidea(Baill.) Webster & Euphorbiaceae & Tree & $\begin{array}{l}\text { Free areas, } \\
\text { forest, dry } \\
\text { outliers }\end{array}$ & Scarce & $\begin{array}{l}\text { Leaves, } \\
\text { branches, } \\
\text { stem, bark, } \\
\text { roots }\end{array}$ & $\begin{array}{c}\text { Green, } \\
\text { dry(Water } \\
\text { boiled ). }\end{array}$ & $\begin{array}{l}\text { Medicinal, } \\
\text { firewood }\end{array}$ \\
\hline 16 & Dongoyaro & AzadirachtaindicaA. Juss & Meliaceae & Tree & $\begin{array}{l}\text { Free areas, } \\
\text { plantation }\end{array}$ & Abundant & $\begin{array}{l}\text { Leaves, } \\
\text { branches, } \\
\text { stem }\end{array}$ & $\begin{array}{c}\text { Green, } \\
\text { dry(Water } \\
\text { boiled ). }\end{array}$ & $\begin{array}{l}\text { Medicinal, } \\
\text { firewood }\end{array}$ \\
\hline 17 & Atare & AfromomumeleguataLindl. & Zingiberaceae & Shrub & $\begin{array}{l}\text { Free areas, } \\
\quad \text { forest }\end{array}$ & Abundant & Fruits & $\begin{array}{c}\text { Green, } \\
\text { dry(Water } \\
\text { boiled ). }\end{array}$ & Medicinal \\
\hline 18 & IgiFrutu & TerminaliacatappaLinn & Combretaceae & Tree & $\begin{array}{l}\text { Free areas, } \\
\quad \text { forest }\end{array}$ & Abundant & $\begin{array}{c}\text { Leaves, fruit, } \\
\text { branches, } \\
\text { stem }\end{array}$ & $\begin{array}{c}\text { Green, } \\
\text { dry(Water } \\
\text { boiled ). }\end{array}$ & $\begin{array}{c}\text { Fruit, } \\
\text { medicinal, } \\
\text { firewood }\end{array}$ \\
\hline 19 & Apa & Afzeliaafricana (Smith) Sm. & $\begin{array}{l}\text { Leguminosae Sub: } \\
\text { Caesalpinioideae }\end{array}$ & Tree & $\begin{array}{l}\text { Forest area, } \\
\text { forest }\end{array}$ & Scarce & $\begin{array}{l}\text { Branches, } \\
\text { stem, bark, } \\
\text { root }\end{array}$ & $\begin{array}{c}\text { Green, } \\
\text { dry(Powder) }\end{array}$ & $\begin{array}{l}\text { Medicinal, } \\
\text { firewood }\end{array}$ \\
\hline 20 & Oboo & Erythrophleumsuaveolens(Gull. and Perr.) & $\begin{array}{l}\text { LeguminosaeSub: } \\
\text { Caesalpinioideae }\end{array}$ & Tree & Forest & Scarce & $\begin{array}{l}\text { Leaves, } \\
\text { branches, } \\
\text { stem, bark, } \\
\text { root }\end{array}$ & $\begin{array}{c}\text { Green, } \\
\text { dry(Water } \\
\text { boiled ). }\end{array}$ & $\begin{array}{l}\text { Medicinal, } \\
\text { firewood }\end{array}$ \\
\hline 21 & Asofeyeje & RauvolfiavomitriaAfzel & Apocynaceae & Tree & $\begin{array}{l}\text { Free areas, } \\
\text { forest }\end{array}$ & Abundant & $\begin{array}{l}\text { Leaves, fruit, } \\
\text { bark, root }\end{array}$ & $\begin{array}{c}\text { Green, } \\
\text { dry(Powder) }\end{array}$ & Medicinal \\
\hline 22 & Omo & CordiamilleniiBak. & Bignoniaceae & Tree & $\begin{array}{l}\text { Free areas, } \\
\quad \text { forest }\end{array}$ & Scarce & $\begin{array}{l}\text { Leaves, } \\
\text { branches, } \\
\text { stem }\end{array}$ & $\begin{array}{c}\text { Green, } \\
\text { dry(Water } \\
\text { boiled ). }\end{array}$ & $\begin{array}{l}\text { Medicinal, } \\
\text { firewood }\end{array}$ \\
\hline 23 & Ewuro & Vernoniaamygdalina(Schreb) Del. & Asteraceae & Tree & $\begin{array}{l}\text { Free areas, } \\
\text { forest }\end{array}$ & Abundant & $\begin{array}{c}\text { Leaves, } \\
\text { branches, } \\
\text { bark, root }\end{array}$ & $\begin{array}{c}\text { Green, } \\
\text { dry(Juice) }\end{array}$ & $\begin{array}{l}\text { Medicinal, } \\
\text { chew-stick }\end{array}$ \\
\hline 24 & Ope & ElaeisguinensisG. Don. & Palmae & Palm Tree & $\begin{array}{c}\text { Swampy } \\
\text { areas, forest, }\end{array}$ & Abundant & $\begin{array}{l}\text { Frond, } \\
\text { exudate, } \\
\text { bark }\end{array}$ & $\begin{array}{c}\text { Green, } \\
\text { dry(Water } \\
\text { boiled ). }\end{array}$ & $\begin{array}{c}\text { Basket, palm } \\
\text { wine, }\end{array}$ \\
\hline 25 & Iya & DanielliaoliveriRolfe & $\begin{array}{c}\text { Leguminosae Sub: } \\
\text { Caesalpinioideae }\end{array}$ & Tree & $\begin{array}{c}\text { Savannah } \\
\text { forest, re- } \\
\text { growth }\end{array}$ & Abundant & $\begin{array}{l}\text { Branches, } \\
\text { stem, bark, } \\
\text { root }\end{array}$ & $\begin{array}{l}\text { Green, } \\
\text { dry(Powder, } \\
\text { Juice) }\end{array}$ & $\begin{array}{l}\text { Firewood, } \\
\text { medicinal }\end{array}$ \\
\hline
\end{tabular}


Journal of Tumor Medicine \& Prevention

\begin{tabular}{|c|c|c|c|c|c|c|c|c|c|}
\hline 26 & Ataile & ZingiberofficinaleRossae. & Zingiberaceae & Herb & $\begin{array}{l}\text { Free areas, } \\
\text { forest }\end{array}$ & Abundant & Rhizome & $\begin{array}{c}\text { Green, } \\
\text { dry(Powder) }\end{array}$ & Medicinal \\
\hline 27 & Ayan & DistemonanthusbenthamianusBenth & $\begin{array}{l}\text { LeguminosaeSub: } \\
\text { Caesalpinioideae }\end{array}$ & Tree & Forest & Abundant & $\begin{array}{l}\text { Leaves, } \\
\text { branches, } \\
\text { stem, bark, } \\
\text { root }\end{array}$ & $\begin{array}{c}\text { Green, } \\
\text { dry(Water } \\
\text { boiled ). }\end{array}$ & $\begin{array}{c}\text { Firewood, } \\
\text { chew stick } \\
\text { medicinal }\end{array}$ \\
\hline 28 & Osankotu & Sidaacuta & Malraceae & Herb & $\begin{array}{c}\text { Forest } \backslash \text { wild, } \\
\text { cultivate }\end{array}$ & Abundant & $\begin{array}{c}\text { Leaves, } \\
\text { branches, } \\
\text { stem, root }\end{array}$ & $\begin{array}{c}\text { Green, } \\
\text { dry(Water } \\
\text { boiled ). }\end{array}$ & Medicinal \\
\hline 29 & Tana'poso & Mirabilis nyctaginea & Nyctaginaceae & Herb & $\begin{array}{c}\text { Forest \wild, } \\
\text { cultivate }\end{array}$ & Abundant & $\begin{array}{c}\text { Leaves, } \\
\text { branches, } \\
\text { stem, root }\end{array}$ & $\begin{array}{c}\text { Green, } \\
\text { dry(Powder) }\end{array}$ & Medicinal \\
\hline 30 & Orin Ata & Zanthoxylumzanthoxyloides & Rutaceae & Herb & $\begin{array}{c}\text { Forest } \backslash \text { wild, } \\
\text { cultivate }\end{array}$ & Abundant & $\begin{array}{l}\text { Branches, } \\
\text { stem, bark, } \\
\text { root }\end{array}$ & $\begin{array}{c}\text { Green, } \\
\text { dry(Powder) }\end{array}$ & $\begin{array}{r}\text { Medicinal } \\
\text { chew stick }\end{array}$ \\
\hline \multirow{6}{*}{31} & & Agerantumconyzoides & & & Wild & & $\begin{array}{c}\text { Leaves, } \\
\text { branches, } \\
\text { stem, root }\end{array}$ & $\begin{array}{c}\text { Green, } \\
\text { dry(Juice) }\end{array}$ & Medicinal \\
\hline & Imiesu & & Compositae & Shrub & & Abundant & & & Insecticide \\
\hline & & & & & & & & & Animal Feed \\
\hline & & & & & & & Leaves & $\begin{array}{c}\text { Green, } \\
\text { dry(Powder) }\end{array}$ & \\
\hline & & Allium sativum Linn & & & & & & & Medicinal \\
\hline & & & & & $\begin{array}{c}\text { Forest } \backslash \text { wild, } \\
\text { cultivate }\end{array}$ & & & & \\
\hline \multirow{4}{*}{32} & Ayu & & Liliaceae & Rhizome & & Abundant & & $\begin{array}{c}\text { Green, } \\
\text { dry(Powder) }\end{array}$ & \\
\hline & & Helianthus annuus & & & & & Leaves, stem, & & \\
\hline & & Securinegavirosa & & & $\begin{array}{c}\text { Forest/wild, } \\
\text { cultivate }\end{array}$ & & & $\begin{array}{c}\text { Green dry } \\
\text { (Water boiled). }\end{array}$ & Medicinal \\
\hline & Sun & & & & & & & & \\
\hline \multirow[t]{2}{*}{33} & Flower & & Asteraceae & Shrub & $\begin{array}{c}\text { Forest } \backslash \text { wild, } \\
\text { cultivate }\end{array}$ & Abundant & $\begin{array}{c}\text { Leaves, } \\
\text { stems, root }\end{array}$ & $\begin{array}{c}\text { Green, } \\
\operatorname{dry(Lotion)}\end{array}$ & \\
\hline & & & & & & & & Juice & Medicinal \\
\hline \multirow{4}{*}{34} & $\begin{array}{c}\text { Ewe } \\
\text { Akintola }\end{array}$ & Vitellariaparadoxa & Euphorbiaceae & & $\begin{array}{c}\text { Forest } \backslash \text { wild, } \\
\text { cultivate }\end{array}$ & & Fruit & & \\
\hline & & Saccharum & & Shrub & $\begin{array}{c}\text { Forest/wild, } \\
\text { cultivate }\end{array}$ & Abundant & & & \\
\hline & & officinarum & & & $\begin{array}{c}\text { Forest \wild, } \\
\text { cultivate }\end{array}$ & & $\begin{array}{c}\text { Leaves, } \\
\text { stems, roots }\end{array}$ & $\begin{array}{c}\text { Green, } \\
\text { dry(Powder) }\end{array}$ & Medicinal \\
\hline & Ori & & & & $\begin{array}{c}\text { Forest } \backslash \text { wild, } \\
\text { cultivate }\end{array}$ & & & $\begin{array}{c}\text { Green, } \\
\text { dry(Powder) }\end{array}$ & \\
\hline \multirow[t]{2}{*}{35} & & Piper guineensis & Sapotaceae & Tree & & Abundant & $\begin{array}{c}\text { Leaves, } \\
\text { stems, roots, } \\
\text { fruits }\end{array}$ & & Medicinal \\
\hline & Ireke & Garcinia koli & & & & & $\begin{array}{l}\text { Fruits, } \\
\text { Leaves }\end{array}$ & & \\
\hline \multirow{3}{*}{36} & Kanafuru & & Poaceae & Shrub & & Abundant & & & Medicinal \\
\hline & & & & & & & & & \\
\hline & & & & & & & & & Food \\
\hline 37 & Orogbo & & Piperaceae & Shrub & & Abundant & & & Medicinal \\
\hline \multirow{2}{*}{38} & & & Guttiferae & Tree & & & & & Food \\
\hline & & & & & & Abundant & & & \\
\hline
\end{tabular}




\section{Journal of Tumor Medicine \& Prevention}

Thirty eight species of Medicinal Plants were identified from the information supplied by the TMPs. Table 1 shows the distribution of the species in relation to the source, availability status, parts of the plant used, form of the plant used, products and the species regeneration in the study area. The life forms of these plants Table 2 shows that the trees constituted the highest number (66\%), followed by shrubs $(20 \%)$, herb (11\%) and rhizome (3\%) In all, the family Leguminosae was dominant with 4 species. This was followed by Annonaceae, Anacardiaceae Euphorbiaceae, and Caesalpinioideae (3 species each).The existence of other plant families in Table 3 demonstrates the rich forest diversity in Southwest Nigeria. This also shows the dynamism in ecosystem maintenance. A number of them also serve economic purposes and are consumed as food in one way or the other. Some of these include: Anacardium occidentalis, A, Mangiferaindica, Musa sapientum, Citrus medica, Vernoniaamygdalina, etc.

Table 1 shows that majority of the TMPs source their medicinal plants from free areas and rarely cultivate them. Table 1 shows that some of the plants are already scarce and species regeneration is by wilding. According to the reports by Gbile et al. [13] and Oguntala et al. [14] the Nigerian ecosystems are at greater risk of extinction if urgent attention is not given to the cultivation of medicinal plants. Table 1 shows that $90 \%$ of the TMPs use the whole plant for treatment that is, they make use of the fruits, stems, barks and leaves at the same time. Table 1 also shows that the forest products used for the treatment of cancer are multipurpose; they are used as firewood, medicine, foods, chewing sticks and animal feeds (Agerantum conyzoides). This corroborate the works of Adekunle [15].

Table 2: The form and method of usage by the traditional medicine practitioners in the treatment of cancer.

\begin{tabular}{|c|c|c|c|c|}
\hline Name of Plant & Species & Form Used & Method of Usage & $\begin{array}{l}\text { No. of Times } \\
\text { Taken }\end{array}$ \\
\hline \multirow[t]{2}{*}{ Eru } & Xylopia aethiopica & \multirow{2}{*}{ Fresh and dry forms } & \multirow{2}{*}{$\begin{array}{l}\text { By boiling in water for } \\
\text { drinking }\end{array}$} & \multirow{2}{*}{ 2ce.Daily } \\
\hline & (Dunal) A. Rich & & & \\
\hline Oganwo & KhayaivorensisA. Chev. & Dry & $\begin{array}{l}\text { By boiling in water for } \\
\text { drinking }\end{array}$ & 3ce. Daily \\
\hline Mango & MagniferaindicaLinn. & Green, fresh and dry. & Juicing with coldwater & 2ce.Daily \\
\hline Kaju & AnacardiumoccidentalisLinn & Green, dry & $\begin{array}{l}\text { By boiling in water for } \\
\text { drinking }\end{array}$ & 3ce. Daily \\
\hline Iyeye & SpondiasmombinLinn. & Green, dry. & $\begin{array}{l}\text { By boiling in water for } \\
\text { drinking }\end{array}$ & 3ce. Daily \\
\hline Abo & AnnonasenegalensisPers & Green, dry & $\begin{array}{l}\text { By boiling in water for } \\
\text { drinking and bathing }\end{array}$ & 3ce. Daily \\
\hline \multirow[t]{2}{*}{ Ahun } & Alstoniaboonei & \multirow{2}{*}{ Green, dry } & \multirow{2}{*}{$\begin{array}{l}\text { By boiling in water for } \\
\text { bathing }\end{array}$} & \multirow{2}{*}{ 2ce.Daily } \\
\hline & De Wild & & & \\
\hline Osanwewe & Citrus medicaLinn. & Green, dry. & By boiling in water, Juice & 2ce.Daily \\
\hline Oruwo & MorindalucidaBenth. & Green, dry. & $\begin{array}{l}\text { By boiling in water, Cold } \\
\text { water squeezed }\end{array}$ & 2ce.Daily \\
\hline Oori-nla & VitexdonianaSweet & Green & $\begin{array}{l}\text { By boiling in water for } \\
\text { drinking }\end{array}$ & 3ce. Daily \\
\hline Osopupa & EnantiachloranthaOliv. & Green, dry & $\begin{array}{l}\text { By boiling in water, } \\
\text { soaking in cold water }\end{array}$ & 2ce.Daily \\
\hline Owu-elepa & PiliostigmathonningiMilne Redhead & $\begin{array}{l}\text { Green, dry (Water } \\
\text { boiled). }\end{array}$ & $\begin{array}{l}\text { By boiling in water for } \\
\text { drinking }\end{array}$ & 3ce. Daily \\
\hline Putu & Ricinodendronheudelotii(Baill) Heckel & Green, dry & soaking in cold water & 3ce. Daily \\
\hline Opoto & FicussurForssk. & Green, dry. & $\begin{array}{l}\text { By boiling in water for } \\
\text { drinking }\end{array}$ & Weekly wash \\
\hline Asasa & Margaritariadiscoidea(Baill.) Webster & Green, dry & $\begin{array}{l}\text { By boiling in water for } \\
\text { drinking }\end{array}$ & 2ce. Daily \\
\hline Dongoyaro & AzadirachtaindicaA.Juss & Green, dry & $\begin{array}{l}\text { By boiling in water for } \\
\text { drinking and bathing }\end{array}$ & 2ce.Daily \\
\hline Atare & AfromomumeleguataLindl. & Green, dry & $\begin{array}{l}\text { By boiling in water, mixing } \\
\text { with pap. }\end{array}$ & 2ce.Daily \\
\hline IgiFrutu & TerminaliacatappaLinn & Green, dry & $\begin{array}{l}\text { Ground, boiling in water } \\
\text { for drinking and bathing }\end{array}$ & 2ce.Daily \\
\hline Apa & Afzeliaafricana(Smith) Sm. & Green, dry & $\begin{array}{l}\text { By boiling in water for } \\
\text { drinking and bathing }\end{array}$ & Weekly Wash \\
\hline Oboo & $\begin{array}{c}\text { Erythrophleumsuaveolens(Gull. and } \\
\text { Perr.) }\end{array}$ & Green, dry & $\begin{array}{l}\text { By boiling in water for } \\
\text { drinking and bathing }\end{array}$ & 2ce.Daily \\
\hline
\end{tabular}


Journal of Tumor Medicine \& Prevention

\begin{tabular}{|c|c|c|c|c|}
\hline Asofeyeje & RauvolfiavomitriaAfzel & Green, dry & $\begin{array}{c}\text { By boiling in water for } \\
\text { drinking }\end{array}$ & 2ce.Daily \\
\hline Omo & CordiamilleniiBak. & Green, dry & $\begin{array}{c}\text { By boiling in water for } \\
\text { drinking }\end{array}$ & 2ce.Daily \\
\hline Ewuro & Vernoniaamygdalina(Schreb) Del. & Green, dry & By boiling in water, Juicing & Once Daily \\
\hline Ope & ElaeisguinensisG. Don. & Green, dry & $\begin{array}{c}\text { By boiling in water for } \\
\text { drinking }\end{array}$ & 2ce.Daily \\
\hline Iya & DanielliaoliveriRolfe & Green, dry & $\begin{array}{c}\text { By boiling in water for } \\
\text { drinking }\end{array}$ & 2ce.Daily \\
\hline Ataile & ZingiberofficinaleRossae. & Green, dry & $\begin{array}{c}\text { By boiling in water for } \\
\text { drinking }\end{array}$ & 2ce.Daily \\
\hline Ayan & DistemonanthusbenthamianusBenth & Green, dry & Heating & Weekly Wash \\
\hline Broom weed & Sidaacuta & Green, dry & By boiling in water, Juicing & 2ce Daily \\
\hline Tana'poso & Mirabilis nyctaginea & Green, dry & $\begin{array}{c}\text { By boiling in water for } \\
\text { drinking }\end{array}$ & 2ce.Daily \\
\hline Fagara & Zanthoxylumzanthoxyloides & Green, dry & $\begin{array}{l}\text { By boiling in water for } \\
\text { drinking }\end{array}$ & 2ce.Daily \\
\hline Goat Weed & Agerantumconyzoides & Green, dry & $\begin{array}{l}\text { By boiling in water, Juicing } \\
\text { for drinking }\end{array}$ & 2ce.Daily \\
\hline Garlic & Allium sativum Linn & Green, dry & $\begin{array}{c}\text { By boiling in water for } \\
\text { drinking }\end{array}$ & 2ce.Daily \\
\hline Sun & Helianthus annuus & Green, dry & $\begin{array}{c}\text { By boiling in water for } \\
\text { drinking }\end{array}$ & 3ce. Weekly \\
\hline \multicolumn{5}{|l|}{ Flower } \\
\hline Bush Weed & Securinega virosa & Green, dry & $\begin{array}{l}\text { By boiling in water for } \\
\text { drinking and bathing }\end{array}$ & 2ce.Daily \\
\hline African Shea Butter & Vitellaria paradoxa & Green & $\begin{array}{l}\text { Processed into lotion to } \\
\text { rub on affected parts of } \\
\text { the body }\end{array}$ & 2ce.Daily \\
\hline \multirow[t]{2}{*}{ Sugar Cane } & & Fresh, Green & & \\
\hline & Saccharum offinarum & & Juice & 2ce.Daily \\
\hline African pepper & Piper guineeensis & Green, dry & $\begin{array}{l}\text { Adjunct to other } \\
\text { preparation }\end{array}$ & 2ce.Daily \\
\hline Bitter Kola & Garcinia koli & Green, dry, wet form & $\begin{array}{l}\text { By boiling in water and } \\
\text { chewing }\end{array}$ & 2ce.Daily \\
\hline
\end{tabular}

Table 2 projects the second objective of this work, it shows that $90 \%$ of the TMPs use the green and dry forms of the forest products; afterwards they use water to soak or boil them. Also, using water the TMPs make juices from plants like Citrus medica, Morinda lucida, Vernonia amygdalina, Sida acuta and Agerantum conyzoides. Table 2 shows that $65 \%$ of the TMPs administer their medications twice daily while $23 \%$ of the TMPs adopt the thrice daily dosage. This helps to ensure frequent interactions and effective communication between the TMPs and their clients unlike the orthodox physicians. This was also reported by Adodo [16]. Weekly wash is employed by $14 \%$ of the TMPs $[17,18]$.

\section{Inferential statistics results for TMPs in Southwest Nigeria}

Inferential Statistics is used to further achieve objectives three and four. (Table 3 ) is the result of the regression analysis showing the relationship between the profit of the Traditional Medicine Practitioners (TMPs) and their demographic data.

Three (3) functional forms of production model including linear, semi-log and Cobb-Douglas (double-log) functions were fitted for the regression analysis. This was done to select the function which gave the result with the best fit. The estimated functions were evaluated in terms of the statistical significance of the coefficient of multiple determinations (R2) as indicated by $\mathrm{F}$ value, the significance of the coefficients and the magnitude of the standard errors. The R2 is the coefficient of multiple determinations which measures the extent to which the variation in the dependent variable is explained by the explanatory variables. The F-value measures the goodness of fit of the model. Based on these statistical and economic criteria, Cobb-Douglas functional form was selected as the lead equation. The coefficient of multiple determinations (R2) obtained for the Cobb-Douglass, that is, 0.437 shows that $43.7 \%$ of the variation in the profit of the TMPs was explained by the included explanatory variables, while the remaining $56.3 \%$ unexplained 


\section{Journal of Tumor Medicine \& Prevention}

was due to the variables not included in the model which was the error term. Number of patients received, total cost of production, age of the practitioners and their years of experience are the significant factors influencing the profit of the practitioners;

Table 3: Regression analysis result to determine demographic factors that affect the profit of the traditional medicine practitioners.

\begin{tabular}{|c|c|c|c|}
\hline Variables & Linear Model & Semi-log Model & Double log Model \\
\hline \multirow{2}{*}{ (Constant) } & -191633.751 & $-6120497.800^{* * *}$ & $3.015^{* * *}$ \\
\hline & $(-0.863)$ & $(-7.560)$ & -7.52 \\
\hline \multirow{2}{*}{ Number of Patients Received } & $5668.860^{* *}$ & $1.154^{*}$ & $0.102^{* *}$ \\
\hline & -2.046 & -1.671 & -2.218 \\
\hline \multirow{2}{*}{ Total Cost of Production } & $0.781^{* * *}$ & $724844.917^{* * *}$ & $0.321^{* * *}$ \\
\hline & -3.659 & -5.356 & -4.627 \\
\hline \multirow{2}{*}{ Age } & $12712.758^{* * *}$ & $1351390.068^{* * *}$ & $0.614^{* * *}$ \\
\hline & -2.77 & -3.144 & -2.954 \\
\hline \multirow[t]{2}{*}{ Years of Experience } & $17349.115^{* *}$ & $821488.191^{* *}$ & $1.134^{*}$ \\
\hline & -2.108 & -2.373 & -1.837 \\
\hline \multirow[t]{2}{*}{ State of Origin } & 0.989 & 0.976 & 1.052 \\
\hline & $(-0.151)$ & $(-0.335)$ & -0.689 \\
\hline \multirow[t]{2}{*}{ Occupation } & 1.041 & 1.03 & 1.015 \\
\hline & -0.559 & -0.415 & -0.219 \\
\hline \multirow[t]{2}{*}{ Gender } & 1.048 & 1.022 & 1.036 \\
\hline & -0.647 & -0.307 & -0.5 \\
\hline \multirow[t]{2}{*}{ Marital Status } & 1.073 & 1.091 & 1.094 \\
\hline & -0.969 & -1.177 & -1.268 \\
\hline \multirow[t]{2}{*}{ Religion } & 1.015 & 1.009 & 1.052 \\
\hline & -0.216 & -0.127 & -0.745 \\
\hline \multirow[t]{2}{*}{ Educational Level } & 0.89 & 0.918 & 0.918 \\
\hline & $(-1.643)$ & $(-1.227)$ & $(-1.264)$ \\
\hline $\mathrm{R} 2$ & 0.404 & 0.394 & 0.437 \\
\hline Adjusted R2 & 0.385 & 0.379 & 0.423 \\
\hline F-statistics & 20.717 & 26.622 & 31.841 \\
\hline
\end{tabular}

traditional medicine practitioners.

Dependent Variable: Profit

*** - significant at $1 \%$ level

** - significant at $5 \%$ level

* - significant at $10 \%$ level

Computed t-values in parenthesis

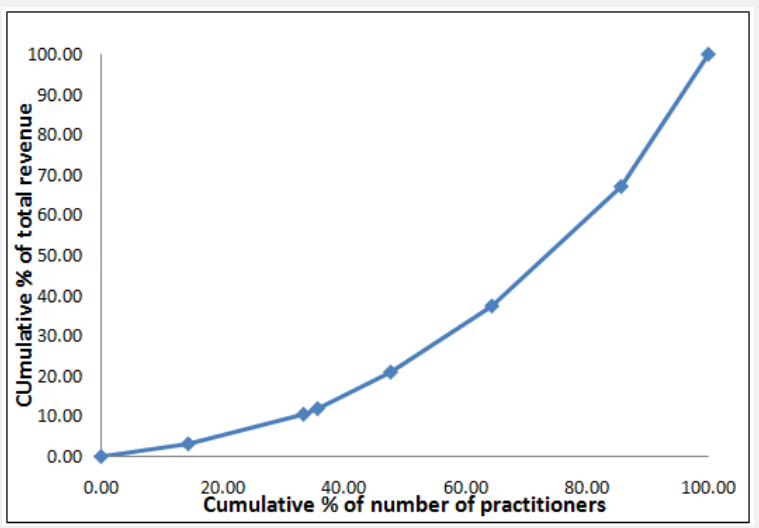

Figure 2: Gini curve. 


\section{Journal of Tumor Medicine \& Prevention}

Table 4 gives the regression analysis result showing the relationship between the profit of the Traditional Medicine Practitioners (TMPs) and some selected variables other than the demographic data of the practitioners. Number of patients per year, duration of treatment, remedy shelf-life, daily application, and time of harvest are shown to have significant positive influence on the profit of the TMPs, which suggests that an increase in these variables would lead to an increase in the profit of the TMPs. However, number of people referred is shown to have a significant negative influence on the profit suggesting that the more that number of people referred by the TMPs the lesser their profits just as it would be expected [19-21].

Table 4: Regression analysis showing relationship between some selected factors and the profits of the Traditional Medicine Practitioners.

\begin{tabular}{|c|c|c|}
\hline Variables & Coefficients & $\mathrm{t}$ - values \\
\hline Constant & -15021498.17 & $2.526^{* *}$ \\
\hline $\begin{array}{c}\text { Number of patients } \\
\text { treated }\end{array}$ & 41022.624 & 1.331 \\
\hline $\begin{array}{c}\text { Number of relatives } \\
\text { affected }\end{array}$ & 5605.058 & 0.051 \\
\hline $\begin{array}{c}\text { Number of people } \\
\text { dead }\end{array}$ & -49103.012 & -0.354 \\
\hline $\begin{array}{c}\text { Number of patients } \\
\text { per year }\end{array}$ & 506016.983 & $2.106^{*}$ \\
\hline $\begin{array}{c}\text { Number of people } \\
\text { referred }\end{array}$ & -531373.962 & $2.514^{* *}$ \\
\hline $\begin{array}{l}\text { Duration of } \\
\text { treatment }\end{array}$ & 1283050.431 & $2.761^{* *}$ \\
\hline Remedy shelf-life & 246731.646 & $2.676^{* *}$ \\
\hline $\begin{array}{l}\text { Method of } \\
\text { production }\end{array}$ & 762933.303 & 1.599 \\
\hline Daily Application & 793581.374 & $2.018^{* *}$ \\
\hline Time of Harvest & 1369993.31 & $3.450^{* * *}$ \\
\hline
\end{tabular}

Dependent Variable: Profit

Traditional Medicine Practitioners.

*** - significant at $1 \%(p<0.01)$ level

** - significant at $5 \%(p<0.05)$ level

* - significant at $10 \%(p<0.1)$ level

(Table 5) is the result of the t-test analysis showing comparison of some selected parameters of the Traditional Medical Practitioners (TMPs) and the Orthodox Medical Practitioners (OMPs). The result shows that there is significant difference in the number of patients recovered, number of deaths recorded, number of referral and the cost of production between the two groups of practitioners with the mean values estimated as follows: number of patients recovered - TMPs (11.92), OMPs (1.99); number of deaths recorded - TMPs (1.75), OMPs (6.61); number of referral - TMPs (3.32), OMPs (8.26) and cost of production - TMPs (N17,246.58), OMPs (N106,750.00). However, the result shows that there is no significant difference in the number of patients treated by the two groups of practitioners. Result of the economic analysis shows minimal competition in the anti-cancer forest product market and a high level of monopoly with a Gini coefficient of 0.83 (Table 6). Net profit was N650, 769.98 (Table 6). Table 6 also shows Rate of Return (280.08\%) and the Rate of Return on Investment (180.08\%) indicating that the TMPs are making profit.

Table 5: $\mathrm{t}$-Tests analysis comparing some selected variables from the Traditional Medicine Practitioners

\begin{tabular}{|c|c|c|c|}
\hline \multirow{2}{*}{ Variables } & TMPs & OMPs & $\begin{array}{c}\mathbf{t} \text { - } \\
\text { values }\end{array}$ \\
\cline { 2 - 3 } (Mean Values) & (Mean Values) & 1.106 \\
\hline $\begin{array}{c}\text { Number of Patients } \\
\text { Treated }\end{array}$ & 16.13 & 19.02 & $6.110^{* *}$ \\
\hline $\begin{array}{c}\text { Number of Patients } \\
\text { Recovered }\end{array}$ & 11.92 & 1.99 & $6.096^{* *}$ \\
\hline $\begin{array}{c}\text { Number of Deaths } \\
\text { Recorded }\end{array}$ & 1.75 & 6.61 & $2.129^{*}$ \\
\hline $\begin{array}{c}\text { Number of Referral } \\
\text { Cost of Treatments }\end{array}$ & 17246.58 & 106750 & $6.530^{* *}$ \\
\hline
\end{tabular}

(TMPs) and the orthodox medical practitioners (OMPs)

** Significant at $1 \%(p<0.01)$ level

* Significant at $5 \%(p<0.05)$ level

Table 6: Annual Average Costs and Returns Analysis

\begin{tabular}{|c|c|}
\hline Item & Value \\
\hline Total Revenue (TR) & 1012142.86 \\
\hline Total Cost (TC) & 361372.88 \\
\hline Net Profit(NP) & 650769.98 \\
\hline Rate of Return (ROR) & $280.08 \%$ \\
\hline $\begin{array}{c}\text { Rate of Return on Investment } \\
\text { (RORI) }\end{array}$ & $180.08 \%$ \\
\hline
\end{tabular}

Table 7: Treatment of identified plants in comparison with Doxorubicin against breast cancer cell line (HS 578T)

\begin{tabular}{|c|c|c|c|c|c|c|}
\hline \multicolumn{7}{|c|}{ After Three Days of Treatment } \\
\hline Con & 0.738 & 0.785 & 0.765 & 0.693 & 0.74525 & 0.0398 \\
\hline \begin{tabular}{c} 
Doxorubicin \\
\hline $\begin{array}{c}\text { Plant1-10ul/ } \\
\mathrm{ml}\end{array}$
\end{tabular} & 0.661 & 0.666 & 0.638 & 0.642 & 0.65175 & 0.01382 \\
\hline $\begin{array}{c}\text { plant1-5ul/ } \\
\mathrm{ml}\end{array}$ & 0.78 & 0.782 & 0.789 & 0.723 & 0.7685 & 0.03058 \\
\hline $\begin{array}{c}\text { Plant1-1ul/ } \\
\mathrm{ml}\end{array}$ & 0.73 & 0.786 & 0.737 & 0.737 & 0.7475 & 0.02588 \\
\hline $\begin{array}{c}\text { Plant2-10ul/ } \\
\mathrm{ml}\end{array}$ & 0.83 & 0.843 & 0.825 & 0.815 & 0.82825 & 0.01164 \\
\hline $\begin{array}{c}\text { plant2-5ul/ } \\
\mathrm{ml}\end{array}$ & 0.818 & 0.802 & 0.853 & 0.829 & 0.8255 & 0.02142 \\
\hline $\begin{array}{c}\text { Plant2-1ul/ } \\
\mathrm{ml}\end{array}$ & 0.8 & 0.793 & 0.809 & 0.799 & 0.80025 & 0.0066 \\
\hline
\end{tabular}


Table 8: Treatment of identified plants in comparison with Doxorubicin against breast cancer cell line (MCF7).

\begin{tabular}{|c|c|c|c|c|c|}
\hline \multicolumn{7}{|c|}{ After Three Days of Treatment } \\
\hline Con & 0.933 & 0.921 & 0.902 & 0.91867 & 0.01563 \\
\hline Doxorubicin & 1.035 & 0.985 & 1.02 & 1.01333 & 0.02566 \\
\hline Plant1-10ul/ml & 1.005 & 0.964 & 0.893 & 0.954 & 0.05667 \\
\hline plant1-5ul/ml & 1.03 & 1.009 & 0.986 & 1.00833 & 0.02201 \\
\hline Plant1-1ul/ml & 1.027 & 0.972 & 0.898 & 0.96567 & 0.06473 \\
\hline Plant2-10ul/ml & 0.944 & 0.889 & 0.934 & 0.92233 & 0.0293 \\
\hline plant2-5ul/ml & 0.877 & 0.918 & 0.861 & 0.88533 & 0.0294 \\
\hline Plant2-1ul/ml & 0.902 & 0.88 & 0.84 & 0.874 & 0.03143 \\
\hline
\end{tabular}

Table 7 shows the test result against cancer cell lines Hs578T while Doxorubicin (a synthetic anticancer drug) was used as the control treatment. Garcinia kola (Bark) did not exhibit significant anticancer effect even at a concentration of $10 \mathrm{u} 1 / \mathrm{m} 1$ while Erythropleum sauveoleons was effective against the cancer cell line at $1 \mathrm{u} 1 / \mathrm{m} 1 . \mathrm{i}$ Table 8 shows the Test result against cancer cell lines MCF7 while Doxorubicin (a synthetic anticancer drug) was used as the control treatment. Garcinia kola (Bark) did not exhibit significant anticancer effect even at a concentration of $10 \mathrm{u} 1 / \mathrm{m} 1$ while Erythropleum sauveoleons was effective against the cancer cell line at $1 \mathrm{u} 1 / \mathrm{m}$

\section{Conclusion}

Forest products are effective in treatment of cancer; therefore in order to achieve the millennium development goals on health; there is need for government to ensure the uniformity of herbal medicine practices. Factors such as, sources and identity of the plant, physical characteristics, chemical constituents, the pharmacological and biological activities of the crude drug and method of preparation, uses and storage, amongst others, need to be identified and documented. This study has justified the importance of plant species in the maintenance of ecosystem and as a source of livelihood for man.

\section{References}

1. Largo M (2014) The Big, Bad Book of Botany: The World's Most Fascinating Flora. Out now from William Morrow, An imprint of Harper Collins Publishers, USA.

2. FAO (1995): Report of the International Expert Consultation on NTFP. Rome, Italy.

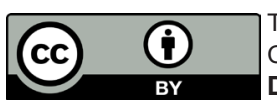

This work is licensed under Creative Commons Attribution 4.0 Licens DOI: 10.19080/JTMP.2017.02.555582
3. FAO (2005): The Support Role: The Use of Forest Resources in other Production Sectors. World Bank Publication, 2004-2005.

4. Marshall E, Newton AC, Schreckenberg K (2003) Commercialization of non-timber products: First steps in analyzing the factors influencing success. International Forestry Review 5(2): 128-137.

5. Okoli RI, Aigbe O, Ohaju Obodo JO, Mensah JK (2007) Medicinal herbs used for managing some common ailments among esan people of edo state, Nigeria. Pak J Nutr 6: 490-496.

6. Olapade EO (2002) The herbs for good health. The $50^{\text {th }}$ Anniversary Lecture of University of Ibadan. Nature cures series 3: 230.

7. Osagie AU, Eka OU (1998) Nutritional qualities of plant foods. Ambik Publishers, Benin City, Nigeria, p. 245.

8. Odugbemi T (2008) A Textbook of Medicinal Plants from Nigeria University of Lagos Press, Lagos, Nigeria, p. 628.

9. IARC (2003) World cancer report 2003. International Agency for Research on Cancer, Lyon, Europe.

10. WHO (2008) The global burden of disease: 2004 update.

11. USAID (2013) Nigeria biodiversity and tropical forests $118 / 119$ assessment. USDA Forest Service Office of International Programs, USA.

12. Gbile ZO, Ola Adams BA, Soladoye MO (1981) Endangered species of the Nigerian flora. Niger J For 8: 14-20.

13. Oguntola AB, Soladoye MO, Ugbogu OA, Fasola TR (1996) A review of endangered tree species of cross river state and environs. Proceedings of the Workshop on Rain Forest of South Eastern Nigeria and South Western Cameroon, Calabar, Nigeria, pp. 120-125.

14. Adekunle AA (2001) Ethnobotanical studies of some medicinal plants from Lagos State, Nigerian Journal of Botany, Nigeria.

15. Adodo A (2003) The Healing Radiance of the Soul: A Guide to Holistic Healing. Agelex Publications, Nigeria.

16. Adodo A (2004) Nature and Power: A Christian Approach to Herbal Medicine. Benedictine Pub Nigeria, Nigeria, p. 289.

17. Adodo A (2005) New Frontiers in African Medicine. Pax Herbal clinic Nig Ltd Ewu Edo State, Nigeria, p. 199.

18. FAO (1987) Forest Products Yearbook.

19. Mallik RM, Panigrahi N (1998) Study of Domestic and Commercial Use of, including Marketing of NTFPs. Scandia consult natura, Sweden, Europe.

20. WHO (2007) Cancer control: knowledge into action: WHO guide for effective programmers: early detection, Geneva, Switzerland.

21. WHO (2006) report on traditional medicine, Geneva, Switzerland.

Your next submission with Juniper Publishers
will reach you the below assets
- Quality Editorial service
- Swift Peer Review
- Reprints availability
- E-prints Service
- Manuscript Podcast for convenient understanding
- Global attainment for your research
- Manuscript accessibility in different formats
( Pdf, E-pub, Full Text, Audio)
- Unceasing customer service
Track the below URL for one-step submission
https://juniperpublishers.com/online-submission.php

